\title{
Winding clusters in percolation on the Torus and the Möbius strip
}

\author{
Running title: "Winding clusters in two-dimensional percolation" \\ Gunnar Pruessner ${ }^{\dagger}$ and Nicholas R. Moloney ${ }^{\ddagger}$
}

October 15, 2003

\begin{abstract}
Using a simulation technique introduced recently, we study winding clusters in percolation on the torus and the Möbius strip for different aspect ratios. The asynchronous parallelization of the simulation makes very large system and sample sizes possible. Our high accuracy results are fully consistent with predictions from conformal field theory. The numerical results for the Möbius strip and the number distribution of winding clusters on the torus await theoretical explanation. To our knowledge, this study is the first of its kind.
\end{abstract}

\section{Introduction}

Since the seminal article of Langlands et al. 1], percolation has enjoyed a renaissance in the last decade. Cardy's result 2 for the probability of a crossing cluster on a rectangle was the first major breakthrough of conformal field theory in critical percolation. Related to this work, Pinson calculated the probability of clusters of particular winding numbers in $[3$, using the partition sum for the torus formulated earlier by di Francesco et al. [4.

In $1996 \mathrm{Hu}$ and Lin showed numerically [5] that the probability of more than one percolating cluster is non-vanishing, which was proven by Aizenman [6] shortly afterwards. In fact, Cardy was able to calculate the asymptotic probability of $n$ distinct, simultaneously crossing clusters [7] exactly. One expects similar behavior for the number of distinct, simultaneously winding clusters on the torus.

In this article we present numerical results for winding clusters on the torus and on the Möbius strip with different aspect ratios, providing strong numerical

\footnotetext{
${ }^{\dagger}$ Email: gunnar.pruessner@physics.org; Address: Department of Mathematics, Imperial College London, 180 Queen's Gate, London SW7 2BZ, UK

${ }^{\ddagger}$ Beit Fellow for Scientific Research; Email: n.moloney@imperial.ac.uk; Address: Condensed Matter Theory, Blackett Laboratory, Imperial College London, Prince Consort Rd, London SW7 2BW, UK
} 
support for the claim of conformal invariance in two dimensional percolation. Apart from the numerical results for $r=1$ by Langlands et al. presented in [3], the only other numerical studies of this kind, to our knowledge, is one by Ziff et al. 8, who measure the probability of a cross topology for very small system sizes $(16 \times 16)$ and different twists on a torus with aspect ratio 1 , and those by Newman and Ziff [9, 10, who measure the probability of winding clusters on a torus with aspect ratio 1 and system sizes up to $256 \times 256$ and varying occupation probability. Also, for the Möbius strip we are only aware of studies on the Ising model [11, 12.

\subsection{Critical percolation on the torus}

We treat site and bond percolation as the two limiting cases of the more general site-bond percolation: Sites are occupied with probability $p^{(\mathrm{s})}$ and bonds are activated with probability $p^{(\mathrm{b})}$. In site percolation all bonds are active, while in bond percolation all sites are occupied. Two sites are connected if the bond between them is active and both sites are occupied. Two sites belong to the same cluster if they are connected by a path along occupied sites and active bonds. On a torus and the Möbius strip, these paths may wind around the lattice. By our convention, $(a, b)$ counts the number of windings in the horizontal and vertical directions, respectively. Vertical and horizontal directions are fixed by the definition of aspect ratio as the vertical circumference (waist) over the horizontal circumference (ignoring the distortion), see Fig. 1

On the torus, it is a topological fact that if $a=0(b=0)$ then the only path which is not homotopic to a point and does not intersect itself has $b=1$

$(a=1)$. There is no topological difference between $(a, b)$ and $(-a,-b)-$ the direction in which these paths are taken is simply inverted. If $a \neq 0$ and $b \neq 0$ then $a$ and $b$ must be relative prime if the path does not intersect itself. Fig. 2 shows an example for $(2,3)$, other examples can be found in [4].

In the following, winding numbers $(a, b)$ will be called "normalized", if $a=1$ for $b=0, b=1$ for $a=0, a$ and $b$ are relative prime if $a \neq 0$ and $b \neq 0$, and $a \geq 0$.

In addition to the above, we also mention the path with the so-called "cross topology" [4. Such a path is produced by intersecting a $(1,0)$ and a $(0,1)$ path. The seam shown in Fig. 1 1 as a dashed line forms a path with a cross topology.

To transfer the notion of winding from paths to clusters, one considers all (topologically different) paths within a cluster. If there is a path with non-zero winding numbers that crosses only paths which are homotopic to a point or have the same winding numbers, the cluster is assigned the winding numbers of the path. If there is no winding path at all, then this cluster itself is said to be homotopic to a point. It transpires that in any other case the cluster contains a cross-topological path and the cluster is then said to have a cross topology itself.

The universal probability to obtain $n$ clusters with winding numbers $(a, b)$ at aspect ratio $r$ is denoted in the following by $\widehat{\mathcal{P}}((a, b), n, r)$. Pinson has derived 

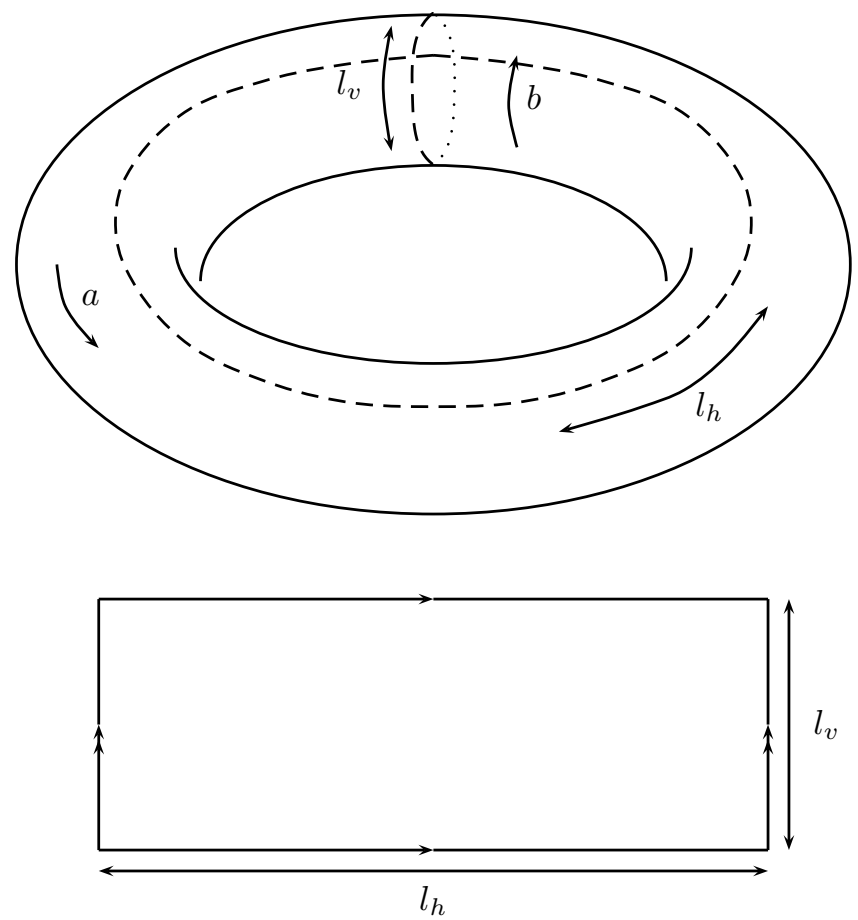

Figure 1: A torus with $r<1$. The winding directions of $(a, b)$ are marked as such and the seam is indicated as dashed line. Below, the "unwrapped" lattice shows the definition of the aspect ratio, $r=l_{v} / l_{h}$.

the formula 3

$$
\begin{aligned}
& \widehat{\mathcal{P}}((a, b), \geq 1, r)=\sum_{l \in \mathbb{Z}} Z_{a 3 l, b 3 l}\left(\frac{2}{3} ; r\right)- \\
& \frac{1}{2} \sum_{l \in \mathbb{Z}} Z_{a(3 l+1), b(3 l+1)}\left(\frac{2}{3} ; r\right)-\frac{1}{2} \sum_{l \in \mathbb{Z}} Z_{a(3 l+2), b(3 l+2)}\left(\frac{2}{3} ; r\right)- \\
& \sum_{l \in \mathbb{Z}} Z_{a 2 l, b 2 l}\left(\frac{2}{3} ; r\right)+\sum_{l \in \mathbb{Z}} Z_{a(2 l+1), b(2 l+1)}\left(\frac{2}{3} ; r\right)
\end{aligned}
$$

where

$$
Z_{m, n}(g ; r)=\frac{\sqrt{g}}{\sqrt{r} \eta^{2}\left(e^{-2 \pi r}\right)} \exp \left(-\pi g\left(\frac{m^{2}}{r}+n^{2} r\right)\right)
$$

and

$$
\widehat{\mathcal{P}}((a, b), \geq n, r)=\sum_{i=n}^{\infty} \widehat{\mathcal{P}}((a, b), i, r) .
$$




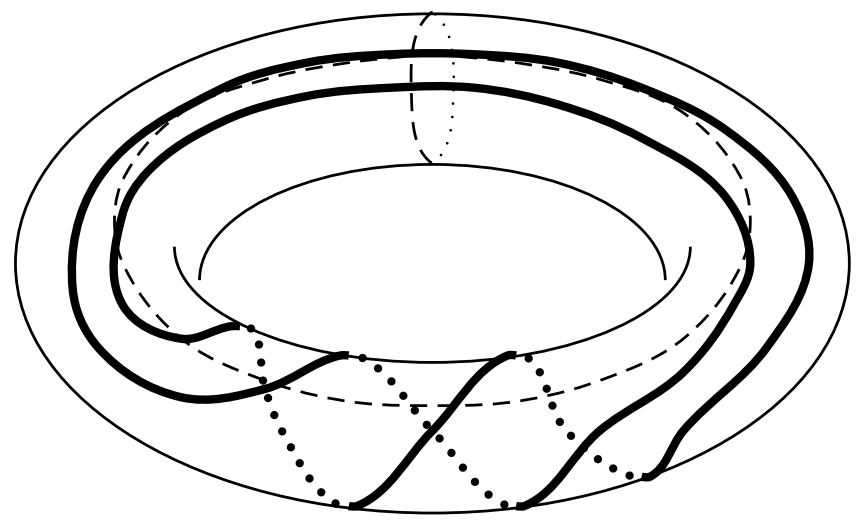

Figure 2: A $(2,3)$ winding on a torus with aspect ratio $r<1$. The seam is shown as a dashed line.

Here $\eta(q)$ is the Dedekind eta function

$$
\eta(q)=q^{1 / 24} \prod_{k=1}^{\infty}\left(1-q^{k}\right) .
$$

Correspondingly, the probability of a cross topology is denoted by $\widehat{\mathcal{P}}(\mathrm{X}, r)$, and the probability that all clusters in a given configuration are homotopic to a point is denoted by $\widehat{\mathcal{P}}(0, r)$. The exact expressions for these probabilities are

$$
\widehat{\mathcal{P}}(\mathrm{X}, r)=\widehat{\mathcal{P}}(0, r)=\frac{1}{2}\left(Z_{c}\left(\frac{8}{3}, 1 ; r\right)-Z_{c}\left(\frac{8}{3}, \frac{1}{2} ; r\right)\right)
$$

with

$$
Z_{c}(g, f ; r)=f \sum_{m, n \in \mathbb{Z}} Z_{f m, f n}(g ; r) .
$$

In the following, we will distinguish exact results from numerical results by putting a hat on all exact quantities. Where necessary, the numerical results will also have an index indicating the system size and a superscript (s) for site percolation and (b) for bond percolation. For example, $\mathcal{P}_{N=3000^{2}}^{(\mathrm{b})}((1,2), 1,9)$ is the fraction of cases in which we observed a single $(1,2)$ cluster in bond percolation with aspect ratio 9 and $3000^{2}$ sites.

The topological considerations in this paper are mainly technically motivated and rather heuristic. For rigorous proofs, we refer to the standard literature [13].

Multiple, distinct clusters with the same winding number can coexist without intersecting 4. This, however, does not apply to a cluster with a cross topology: there can only be one such cluster on a torus. As explained below in detail, winding clusters with incommensurable winding numbers cannot coexist. Thus an entire configuration of the lattice on the torus is characterized by the winding numbers of the winding clusters, if any, and their total number. 
The Möbius strip is a little more complicated in this respect. First of all, since the Möbius strip is a non-orientable surface, a reasonable definition of a spanning cluster connecting one side to the other is not possible, unlike, for example, clusters on a cylinder connecting top and bottom [14, 17. Winding clusters behave rather surprisingly. A single winding cluster, winding around only once, is possible. Meanwhile, if two winding clusters coexist, then at least one of them must have winding number 2 . In general, $n$ winding clusters require at least $n-1$ clusters with winding number 2 . In fact, all winding clusters are fully defined by the total number $a$ of windings alone: If $a$ is even, there are $a / 2$ winding clusters with winding number 2 . If $a$ is odd, there are $(a-1) / 2$ winding clusters with winding number 2 , and 1 cluster with winding number 1 . Fig. 3 shows a Möbius strip with 2 winding paths adding up to a total winding number 3. The aspect ratio of the Möbius strip is defined as the width of the band over the path length along the band, as shown in Fig. [3]
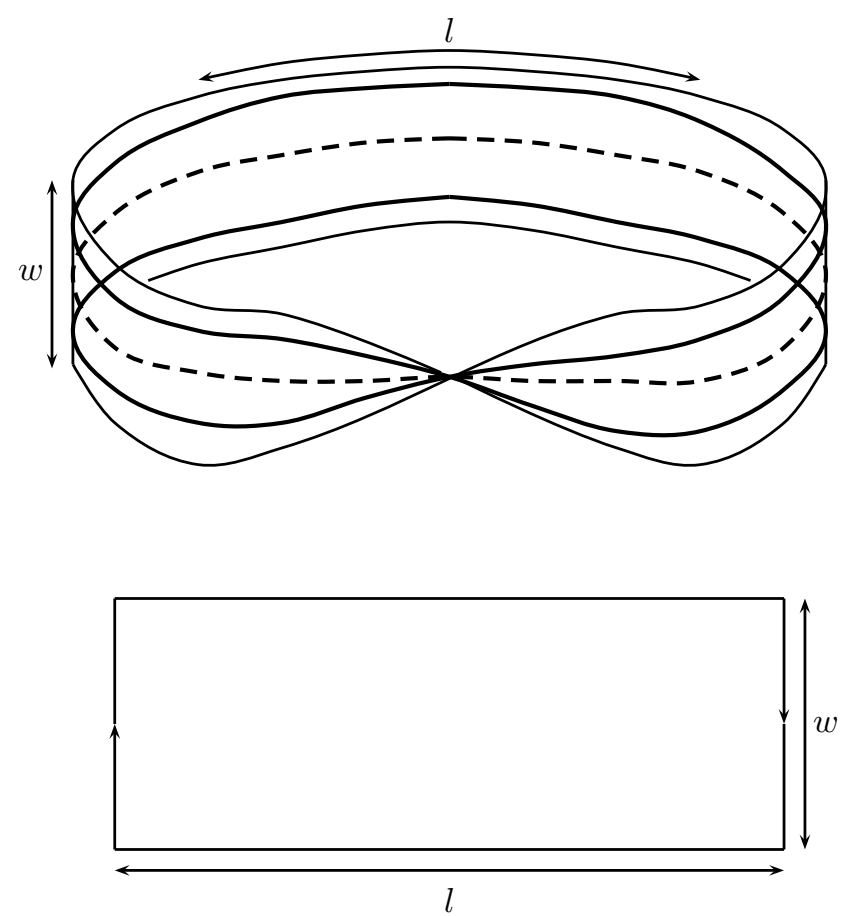

Figure 3: A Möbius strip with two non-intersecting winding clusters, one with winding number 2 (full line) and one with winding number 1 (dashed line). Below, the "unwrapped" lattice shows the definition of the aspect ratio, $r=w / l$.

One might be tempted to extend the study of winding numbers to the Klein bottle. In this case, however, the wrapping around the waist is ill-defined, 
just like for the spanning cluster on the Möbius strip discussed above. The twist in the boundary conditions breaks translational invariance; for example, a winding path, for which the algorithm (see below) would indicate winding numbers $(1,0)$, can be smoothly transformed into a path with winding numbers $(1,1)$. This also makes the Klein bottle fundamentally different from the torus with a twist, which has a fixed "offset" for its winding numbers. Therefore, we did not study the Klein bottle.

\section{Method}

The simulation is based on a method described in detail in [15, which in principle relaxes all the standard constraints in numerical simulations of percolation, such as CPU power, memory, and network capacity. The method is especially suited for calculating cluster size distributions and crossing probabilities. Here we have used the method to calculate probabilities of winding clusters on the torus and the Möbius strip.

The algorithm runs asynchronously across a cluster of computers (slaves), which supply a central node (master) with "patches". These patches are produced by simulating percolation on squares and encoding in the boundary information about all clusters touching the boundary. The data representation is due to Hoshen and Kopelman [16] (HK), and the reduction of the data to the boundary is essentially a form of "Nakanishi label recycling" [17, 18. The patches are "glued together" by the master node to form larger lattices with various boundary conditions, such as periodic boundaries used here. One of the key features of the algorithm is that the same patches can be used for different topologies (such as the torus and the Möbius strip) and aspect ratios. To reduce correlations between different results, patches can be randomly permuted, mirrored and rotated. We have applied this technique for each aspect ratio.

Technical details concerning the method can be found in [15] and, especially regarding the detection of wrapping clusters, in [14. Here we assume that a master node has received a number of square patches of size $L^{2}$, and has (randomly) glued them together to form a lattice with open boundaries of size $N$ and aspect ratio $r$. At this stage, sites are still encoded in HK style: Every site on the border has a pointer attached to it, which points either to its representative or to itself. If it points to itself, the site is called a root site. A representative may or may not point to another representative, so that the pointers form a treelike structure. The algorithm ensures that a tree has exactly one root and that all sites within the same cluster belong to the same tree. Thus, all information about a cluster can be stored at the root site and is accessible from every site belonging to the cluster.

To facilitate the calculation of winding numbers, the HK representation is extended so that each pointer includes a distance, indicating the multiple of $\pi$ from a given site to its representative. The path length from a given site to its root is then given by the sum over all distances along the path in the tree of representatives. Details about this can be found in [14]. 


\section{$2.1 \quad$ Identifying clusters}

When a torus is formed by the master from a rectangular lattice with open boundaries, periodic boundaries are applied across opposite sides of the rectangle. We call these boundaries the "seam" of the torus, which is shown in Fig. 1 The seam itself is a cross topology, so that it necessarily crosses all clusters which are not homotopic to a point. Therefore, all winding clusters are encountered during the gluing procedure and it is only there and then that one has to detect winding numbers. The seam is fastened like a zip that runs along pairs of sites. During this process clusters are detected to be winding and/or merge. If two sites either side of the seam are seen to be part of the same cluster prior to local gluing, because they are connected via another path (which may or may not cross the seam closed so far), a winding path has been detected, provided that the paths from the two sites to the same root differ. After the new path is normalized, two distinct cases need to be distinguished: 1) The cluster so far contains no winding path. In this case, the winding numbers are assigned to the cluster. 2) The cluster is already known to be winding, with winding numbers $(a, b)$. If the new path $\left(a^{\prime}, b^{\prime}\right)$ obeys $\left(a^{\prime}, b^{\prime}\right)=(a, b)$, then the new path "runs parallel" to the old one. If the old and new paths differ, the cluster must have a cross topology: since both paths are normalized, they cannot be multiples of each other, because if there was a $k$ such that $(k a, k b)=\left(a^{\prime}, b^{\prime}\right)$ or $\left(k a^{\prime}, k b^{\prime}\right)=(a, b)$ one of the two paths would not be normalized. If $(a, b)=(1,0)$ then the new path must have $b^{\prime} \neq 0$. Therefore $(a, b)$ intersects $\left(a^{\prime}, b^{\prime}\right)$ thereby providing a "shortcut" and forming a path $(0,1)$. That is, $\left(a^{\prime}, b^{\prime}\right)$ taken forwards and $(1,0)$ taken $a^{\prime}$ times backwards gives $\left(0, b^{\prime}\right)$, which, normalized, is just $(0,1)$ (see below).

The general case is that both paths wind in both directions. They are not multiples, so $a / a^{\prime} \neq b / b^{\prime}$. But then it is possible to find a path of the form $(q, 0)$ with $q \neq 0$, namely by taking $b^{\prime}$ times path $(a, b)$ and $b$ times path $\left(a^{\prime}, b^{\prime}\right)$ backwards, which results in a path $\left(b^{\prime} a-b a^{\prime}, 0\right)$, with $b^{\prime} a-b a^{\prime} \neq 0$. All paths constructed by forward and backward movements may have their clusters deformed in such a way that the resulting path is composed of only forward movements. A path $(q, 0)$ with $|q|>1$ must intersect itself, so that this path will always contain a path $(1,0)$.

The same construction provides a path $(0,1)$. Thus once again we arrive at the cross topology: if a cluster contains two incommensurable paths, then such a cluster has a cross topology.

The recipe above provides a unique way to determine the winding numbers of a single cluster whenever different paths are identified in it. Similar principles apply to the entire configuration: The configuration itself is assigned winding numbers and a counter, keeping track of how often a particular type of winding cluster occurs. A case not mentioned above is that of two clusters, already known to be winding, that are merged across the seam. If the paths have the same normalized winding numbers, the counter is reduced by one. It cannot happen that they do not have the same winding numbers: It is topologically impossible that two clusters are both known to be winding with different winding 
numbers before being merged at a later stage. Thus, if the first winding cluster is found, its winding number is assigned to the entire configuration and the counter is set to one. If a further, distinct cluster is found to be winding, it must wind with the same winding numbers, so that the counter is increased by one. If any cluster is found to have a cross topology, then all clusters will have a cross topology or be homotopic to a point. The cross topology is assigned to the entire configuration and the counter is set to one.

Similar rules can be found for the Möbius strip. As explained above, a single cluster with winding number 1 can coexist with multiple clusters of winding number 2. Any new winding cluster found increases the total winding number of the configuration by its winding number. If two clusters merge, at least one of them must wind twice, reducing the total number of windings by 2 .

\section{Results}

In this section we first list the key parameters of the simulation. Then we discuss the assumptions and estimates of the numerical errors associated with the study. In the following subsection we present the results for the torus which can be compared to Pinson's formulae (11) and (15), asymptotes for which are presented in Section 3.4 In the following subsections, we present the results for the number distribution of multiple winding clusters and the results for the Möbius strip.

In each simulation, we have determined $\mathcal{P}_{N}((a, b), n, r)$ as well as $\mathcal{P}_{N}(0, r)$ and $\mathcal{P}_{N}(\mathrm{X}, r)$ for site and bond percolation at the critical point, i.e. occupation probability $p^{(\mathrm{s})}=0.59274621$ 10 in site percolation and activation probability $p^{(\mathrm{b})}=1 / 2[19$ in bond percolation.

Each simulation, parametrized by the system size $N$ and the type of percolation (site or bond), consists of at least $10^{6}$ realizations. A realization requires 900 quadratic patches of size $L^{2}(L=10,100,1000)$ produced by the slaves, which are joined together by the master, to form a lattice of size $N=900 L^{2}$ and aspect ratio $r$, so that $N=30000^{2}, 3000^{2}, 300^{2}$. The resulting lattice is then "glued" together in different ways to form the different topologies. The 14 different aspect ratios are: $30 / 30,36 / 25,45 / 20,50 / 18,60 / 15,75 / 12,90 / 10$, $100 / 9,150 / 6,180 / 5,225 / 4,300 / 3,450 / 2$ and $900 / 1$. While a torus with aspect ratio $r$ is topologically identical to a torus with aspect ratio $1 / r$, a Möbius strip can be glued along two different borders to form a band either of aspect ratio $r$ or $1 / r$. Thus, 27 different aspect ratios are available for the Möbius strip.

The same 900 patches are used for all different topologies and aspect ratios, but they are randomly permuted, rotated and mirrored before forming a new aspect ratio. The random number generator used is described in [20], and is especially suitable for parallel applications.

The slaves, producing the 900 patches, themselves apply the appropriate boundary conditions, so that the simulation produces of the order of $10^{9}$ samples for the torus and the Möbius strip for $r=1$ and $L=10,100,1000$. We restrict the presentation of these results to special cases. 


\subsection{Numerical errors}

In a numerical simulation an estimate $p$ for the probability of the occurrence of a particular property (such as 2 winding clusters with winding number $(1,3)$ ) is measured as the average of an indicator function $f(\mathcal{C})$ of the configuration $\mathcal{C}$, which is 1 , if the property is found in the configuration and 0 otherwise. As all higher moments of $f$ are $p$, the variance of $p$ is then simply estimated as $p-p^{2}$, so that the variance of the estimator of the probability is estimated as $\left(p-p^{2}\right) /(N-1)$, where $N$ is the number of (independent) realizations.

It is worth mentioning a subtlety which can give rise to a systematic overestimation of the underestimation of the probability and vice versa: If the variance of the estimator for the probability $p$ is estimated from the numerical data, and this data indicates a slightly smaller probability than the exact result, the variance is likewise estimated slightly too small, if $p<1 / 2$. Therefore, the relative numerical deviation of the numerically estimated probability from the exact result will seem slightly too large. This becomes immediately clear if one wants to calculate the relative error for the probability for a configuration which actually never occurred: the estimated probability and the estimated variance both vanish. Moreover, we note that the estimation of the variance itself acquires an increasing error as the probability approaches 0 or 1 .

Vice versa, if the probability $p<1 / 2$ is slightly overestimated from the numerical data, so too is the variance, and therefore the relative error is underestimated. Correspondingly for $p>1 / 2$, where overestimation leads to underestimation of the variance and therefore to overestimation of the relative error and vice versa. However, since the aim of this work is to corroborate analytical work, all errors are based on the numerical results only, wherever possible. Only if the numerics give 0 or 1 is the error based on the analytical result. If the analytical result is not available because of numerical convergence problems, no result is shown.

As mentioned above, the same 900 patches are used multiple times to form rectangular lattices of different aspect ratios. Three different sets of boundary conditions (one for the torus, two for the Möbius strip) are then applied to the same rectangular lattice and the different measurements are taken. Thus, there are correlations which, however, we do not explicitly account for, primarily because we expect them to be very small. In fact, the correction to the error indicated depends on how many measurements are considered simultaneously: Assuming maximum correlations in $n$ measurements, one could divide the sample size by $n$, as if each quantity were based on its own subset of the sample. Accepting the averages calculated from the complete sample as good estimators of the averages calculated from each subsample, this procedure leads to a factor of $\sqrt{n}$ in front of each standard deviation.

In the following we make use of the symmetry of the torus,

$$
\begin{aligned}
\widehat{\mathcal{P}}((a, b), n, r) & =\widehat{\mathcal{P}}\left((b, a), n, r^{-1}\right) \\
\widehat{\mathcal{P}}(0, n, r) & =\widehat{\mathcal{P}}\left(0, n, r^{-1}\right) \\
\widehat{\mathcal{P}}(\mathrm{X}, n, r) & =\widehat{\mathcal{P}}\left(\mathrm{X}, n, r^{-1}\right),
\end{aligned}
$$


i.e. the results of $r$ and $1 / r$ are not independent.

\subsection{Cross topology}

One surprising result in Pinson's paper $[3]$ is that the probability of a cluster with

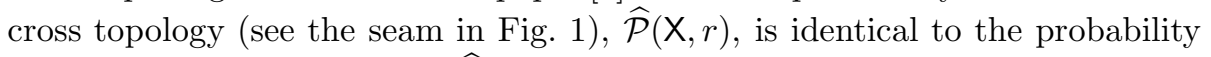
of no winding cluster at all, $\widehat{\mathcal{P}}(0, r)$, i.e. the probability that all clusters are homotopic to a point. This is in perfect agreement with our numerical results: For $N=30000^{2}$ Fig. 4 shows the deviation of $\mathcal{P}_{N}(\mathrm{X}, r)$ and $\mathcal{P}_{N}(0, r)$ from the exact result (5) for site and bond percolation*.

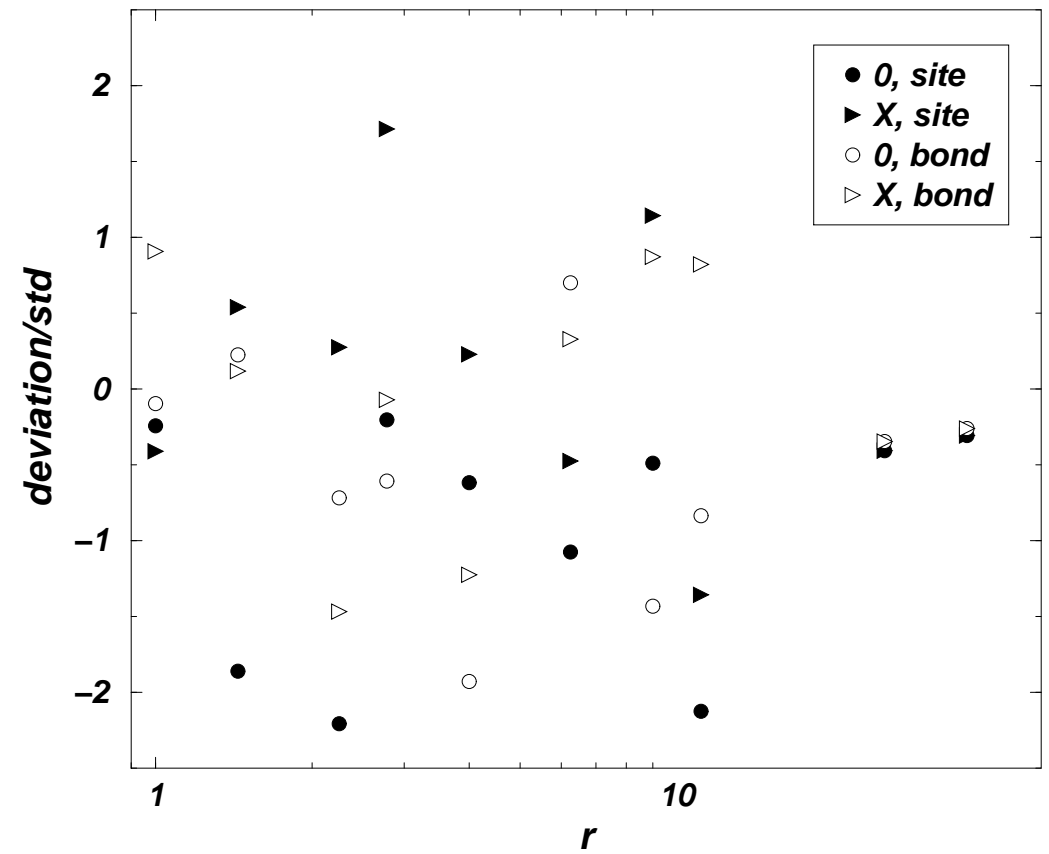

Figure 4: The deviation of $\mathcal{P}_{N=30000^{2}}(\mathrm{X}, r)$ and $\mathcal{P}_{N=30000^{2}}(0, r)$ from $\widehat{\mathcal{P}}(\mathrm{X}, r)$ (see (5)) in units of standard deviations for site (filled symbols) and bond (opaque symbols) percolation.

It is obvious that there can only be one cluster with a cross topology, and that any other cluster must be homotopic to a point, i.e. if a configuration contains a cluster with a cross topology, there is no other non-trivial cluster.

* There seems to be a subtle underestimation of $\widehat{\mathcal{P}}(0, r)$ and an overestimation of $\widehat{\mathcal{P}}(\mathrm{X}, r)$, best seen in the slight segregation of triangles and circles. We are not sure yet what this effect could indicate. It is not present for $N=3000^{2}$ and $N=300^{2}$. 


\subsection{Winding clusters}

Next we investigate the probability of at least one cluster with winding numbers $(a, b)$, the exact expression for which was conjectured by Pinson to be (11). Fig. [5 shows the relative deviation of the numerical results from the exact value for clusters with winding numbers $(1,0),(1,1)$ and $(1,2)$ for $N=30000^{2}$ and bond percolation. The reason why so many points seem to indicate no deviation at all is that the probability of certain types of winding clusters is extremely small. As a result, some rare types were not observed in the simulation and the resulting deviation from the exact result is approximately $\sqrt{p} / \sqrt{N-1}$, i.e. extremely small. Moreover, it should be noted that the numerical error of the evaluation of (11) increases as the probability approaches 0 or 1 .

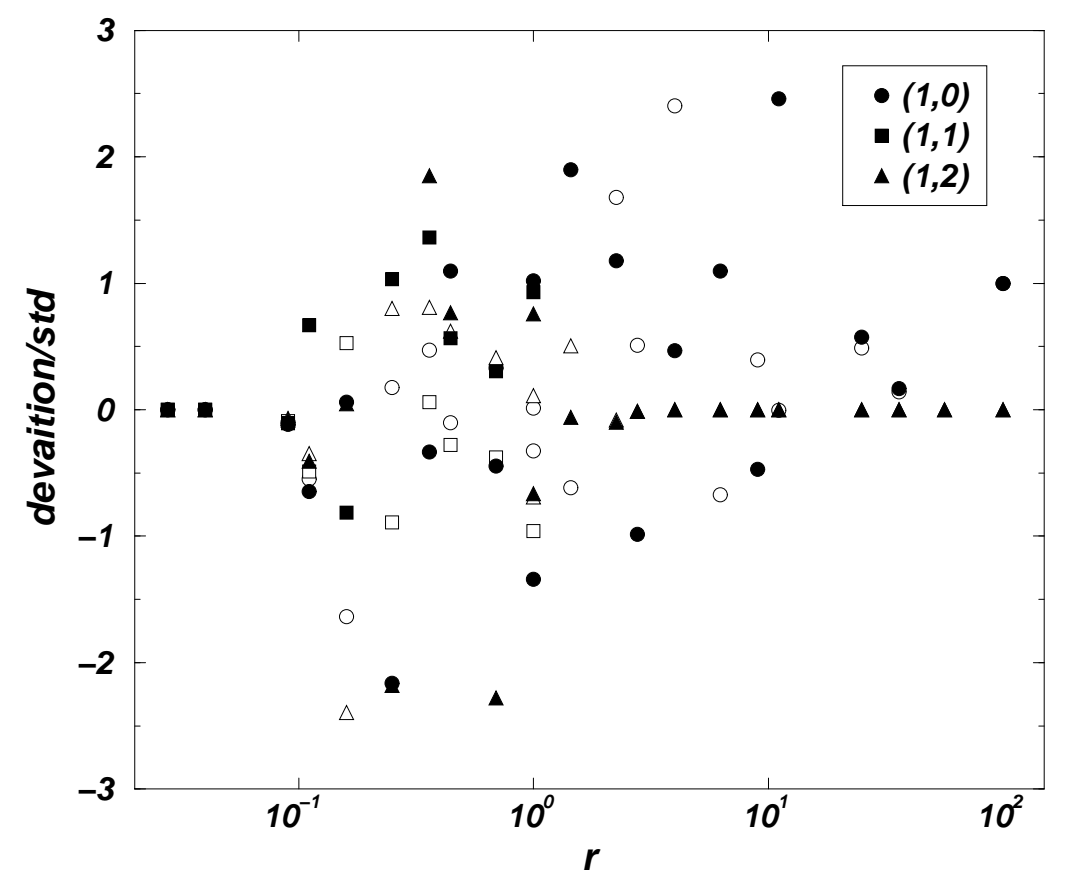

Figure 5: The deviation of $\mathcal{P}_{N=30000^{2}}((a, b), \geq 1, r)$ from $\widehat{\mathcal{P}}((a, b), \geq 1, r)$ (see (11)) in units of standard deviations for $(a, b)=(1,0),(1,1),(1,2)$ and site (filled symbols) and bond (opaque symbols) percolation.

Fig. [6] shows a typical set of data for winding numbers $(1,2)$. It is clear that the probability for a $(1,2)$ winding cannot be symmetric around $r=1$. Above $r=2.0$ it vanishes, because it becomes less and less likely for a cluster to wind around twice as the aspect ratio increases. Correspondingly, as $r$ decreases it becomes less and less likely for a cluster to wind around once in the orthogonal direction. 
All our numerical findings are fully consistent with (11) for winding numbers $(1,0),(1, \pm 1),(1, \pm 2)$ and $(1, \pm 3)$, in site and bond percolation. Other winding numbers occur too rarely to make any firm statements.

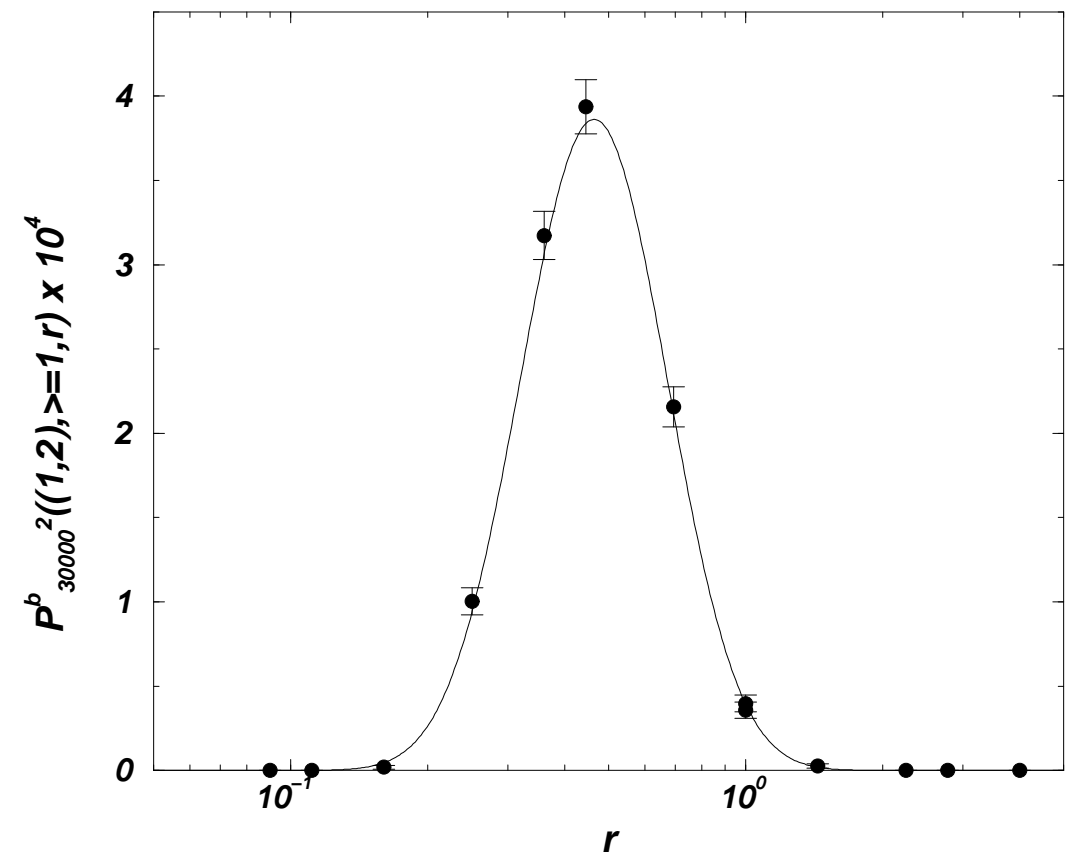

Figure 6: The rescaled probability $\mathcal{P}_{N=30000^{2}}^{(\mathrm{b})}((1,2), \geq 1, r)$ for different aspect ratios. The rescaling factor is $10^{4}$. The straight line shows the analytical result (11).

Percolation on a torus is mirror symmetrical, i.e.

$$
\widehat{\mathcal{P}}((a, b), n, r)=\widehat{\mathcal{P}}((a,-b), n, r)=\widehat{\mathcal{P}}((-a, b), n, r)=\widehat{\mathcal{P}}((-a,-b), n, r) .
$$

While a cluster of type $(a, b)$ is simply identical to a cluster of type $(-a,-b)$, the windings $(a, b)$ and $(a,-b)$ are in fact distinguishable. Since these two quantities have the same probability, their comparison affords a consistency check upon the numerics, independent of any theoretical result or finite size corrections. We have passed this test successfully.

\subsection{Asymptotes}

To discuss numerical results for the asymptotic behavior of multiple winding clusters, we must first extract an appropriate functional form to fit against from (1). A calculation closely related to the following was presented by Ziff for 
the problem of crossing in two-dimensional percolation [21, 22, and subsequently used in [14.

It is convenient to slightly rewrite Eq. (1). Noting that all $Z_{m, n}$ enter (1) in the form $Z_{a k, b k}$, it is reasonable to define

$$
\tilde{Z}((a, b) ; g, r)=\sqrt{\frac{g}{r}} \sum_{l \in \mathbb{Z}} \exp \left(-l^{2} \pi g\left(\frac{a^{2}}{r}+b^{2} r\right)\right),
$$

which is just $\sqrt{g / r} \vartheta_{3}(0, \tau)$, where $\tau=i g\left(a^{2} / r+b^{2} r\right)$ and $\vartheta_{3}$ is Jacobi's theta function [23]. By using transformations of the form $\sum_{l} f_{2 l+1}=\sum_{l} f_{l}-\sum_{l} f_{2 l}$ one has

$$
\begin{aligned}
& \widehat{\mathcal{P}}((a, b), \geq 1, r)=\frac{1}{\eta\left(e^{-2 \pi r}\right)^{2}} \\
& \times\left(\frac{1}{2} \tilde{Z}((a, b) ; 6, r)-\tilde{Z}((a, b) ; 8 / 3, r)+\frac{1}{2} \tilde{Z}((a, b) ; 2 / 3, r)\right),
\end{aligned}
$$

given appropriate convergence of the sums in (8) and (11). For $(a, b)=(1,0)$ Eq. (8) transforms into a Riemann sum with mesh size $1 / \sqrt{r}$, and for large $r$ it converges to a Gaussian integral, i.e. $\lim _{r \rightarrow \infty} \tilde{Z}((a, b) ; g, r)=1$. That, however, leads to $\lim _{r \rightarrow \infty} \exp (\pi r / 6) \times 0$ in (9). This can easily be fixed using the Poisson summation formula (or the properties of $\vartheta_{3}[23]$ ), which gives

$$
\sum_{l \in \mathbb{Z}} e^{-l^{2} u^{2}} u=\sqrt{\pi} \sum_{l \in \mathbb{Z}} e^{-\pi^{2} l^{2} / u^{2}}
$$

and therefore for general $(a, b)$

$$
\tilde{Z}((a, b) ; g, r)=\frac{1}{\sqrt{a^{2}+b^{2} r^{2}}} \sum_{l \in \mathbb{Z}} \exp \left(-\frac{\pi}{g\left(a^{2}+b^{2} r^{2}\right)} l^{2} r\right) .
$$

From the definition of the Dedekind eta function

$$
\eta(\exp (-2 \pi r))=e^{-\frac{\pi r}{12}} \prod_{k=1}^{\infty}\left(1-e^{-2 k \pi r}\right)
$$

one finds the following expansion for large $r$ :

$$
\eta^{-2}(\exp (-2 \pi r))=e^{\pi r / 6}\left(1+2 e^{-2 \pi r}+5 e^{-4 \pi r}+10 e^{-6 \pi r}+\ldots\right) .
$$

Using (11) for the large $r$ expansion of (9) at $(a, b)=(1,0)$

$$
\tilde{Z}((1,0) ; g, r)=1+2 e^{-\frac{\pi r}{g}}+2 e^{-4 \frac{\pi r}{g}}+2 e^{-9 \frac{\pi r}{g}}+2 e^{-16 \frac{\pi r}{g}}+\ldots,
$$

the task boils down to ordering terms:

$$
\widehat{\mathcal{P}}((1,0), \geq 1, r)=1-2 e^{-\frac{5}{24} \pi r}+e^{-\frac{1}{2} \pi r}+2 e^{-2 \pi r}-4 e^{-\frac{53}{24} \pi r}+3 e^{-\frac{5}{2} \pi r} \ldots
$$




\begin{tabular}{l|l|ll|ll}
$n$ & $r$ range & $C^{(\mathrm{s})}((1,0), n)$ & $\alpha((1,0), n)$ & $C^{(\mathrm{b})}((1,0), n)$ & $\alpha((1,0), n)$ \\
\hline 1 & $12 / 75 \ldots 30 / 30$ & $0.811(3)$ & $1.568(3)$ & $0.810(3)$ & $1.566(3)$ \\
2 & $25 / 36 \ldots 60 / 15$ & $0.856(3)$ & $7.771(12)$ & $0.868(4)$ & $7.813(14)$ \\
3 & $36 / 25 \ldots 75 / 12$ & $1.364(10)$ & $19.02(4)$ & $1.368(11)$ & $19.02(5)$ \\
4 & $50 / 18 \ldots 90 / 10$ & $2.05(2)$ & $33.91(8)$ & $2.10(2)$ & $34.05(10)$ \\
5 & $60 / 15 \ldots 100 / 9$ & $3.76(6)$ & $53.94(16)$ & $3.71(7)$ & $53.82(19)$
\end{tabular}

Table 1: Multiple winding clusters $(1,0)$. The numerical results $\mathcal{P}_{N=30000^{2}}((1,0), n, r)$ are fitted within the range indicated against (20).

This approximation has a relative deviation from the exact result of less than $5 \times 10^{-4}$ at $r=1$ and less than $10^{-8}$ at $r=2$.

Using $\widehat{\mathcal{P}}((1,0), \geq 1, r)=\widehat{\mathcal{P}}((0,1), \geq 1,1 / r)$, the corresponding expansion for small $r$ is now based directly on (8). Thus again for large $r$

$$
\widehat{\mathcal{P}}((0,1), \geq 1, r)=\sqrt{\frac{2}{3 r}}\left(e^{-\frac{1}{2} \pi r}-e^{-\frac{5}{2} \pi r}-e^{-\frac{9}{2} \pi r}+4 e^{-\frac{35}{6} \pi r} \ldots\right) .
$$

At $r=1$ the relative error of this approximation is better than $3 \times 10^{-8}$, which improves to about $10^{-15}$ at $r=2$.

Similarly one finds for $\widehat{\mathcal{P}}(\mathrm{X}, r)$

$$
Z_{c}(f, g ; r)=\frac{1}{\eta\left(e^{-2 \pi r}\right)^{2}} \tilde{Z}\left((1,0) ; f^{2} g, r\right) \tilde{Z}\left((1,0) ; 1 /\left(f^{2} g\right), r\right)
$$

which yields together with (13) and (14)

$$
\widehat{\mathcal{P}}(\mathbf{X}, r)=e^{-\frac{5}{24} \pi r}-e^{-\frac{1}{2} \pi r}-2 e^{-2 \pi r}+2 e^{-\frac{53}{24} \pi r}-2 e^{-\frac{5}{2} \pi r} \ldots
$$

again for large $r$. The relative deviation is less than $9 \times 10^{-4}$ at $r=1$ and about $10^{-7}$ at $r=2$.

\subsection{Multiple winding clusters}

Unfortunately, it is not straightforward to extend Cardy's qualitative arguments in the introduction of [7] for the existence of multiple spanning clusters.

Multiple winding clusters with winding numbers other than $(1,0)$ are very rare. In fact, we found

$$
\begin{aligned}
& \mathcal{P}_{N=1000^{2}}^{(\mathrm{s})}((1,1), 2,1)+\mathcal{P}_{N=1000^{2}}^{(\mathrm{s})}((1,-1), 2,1)=1.40(8) \times 10^{-7} \\
& \mathcal{P}_{N=1000^{2}}^{(\mathrm{b})}((1,1), 2,1)+\mathcal{P}_{N=1000^{2}}^{(\mathrm{b})}((1,-1), 2,1)=1.50(10) \times 10^{-7}
\end{aligned}
$$

based on the data produced at the slave nodes. Probabilities for higher multiples and other winding numbers are less than about 1 in $2 \times 10^{9}$.

From what has been said in Section 3.4 (see also Eq. (21)) one might suspect that the probability of $n$ distinct, simultaneously winding clusters with winding number $(1,0)$ behaves in the limit of small $r$ like

$$
\widehat{\mathcal{P}}((1,0), n, r) \approx C((1,0), n) e^{-\alpha((1,0), n) / r} \sqrt{r} .
$$




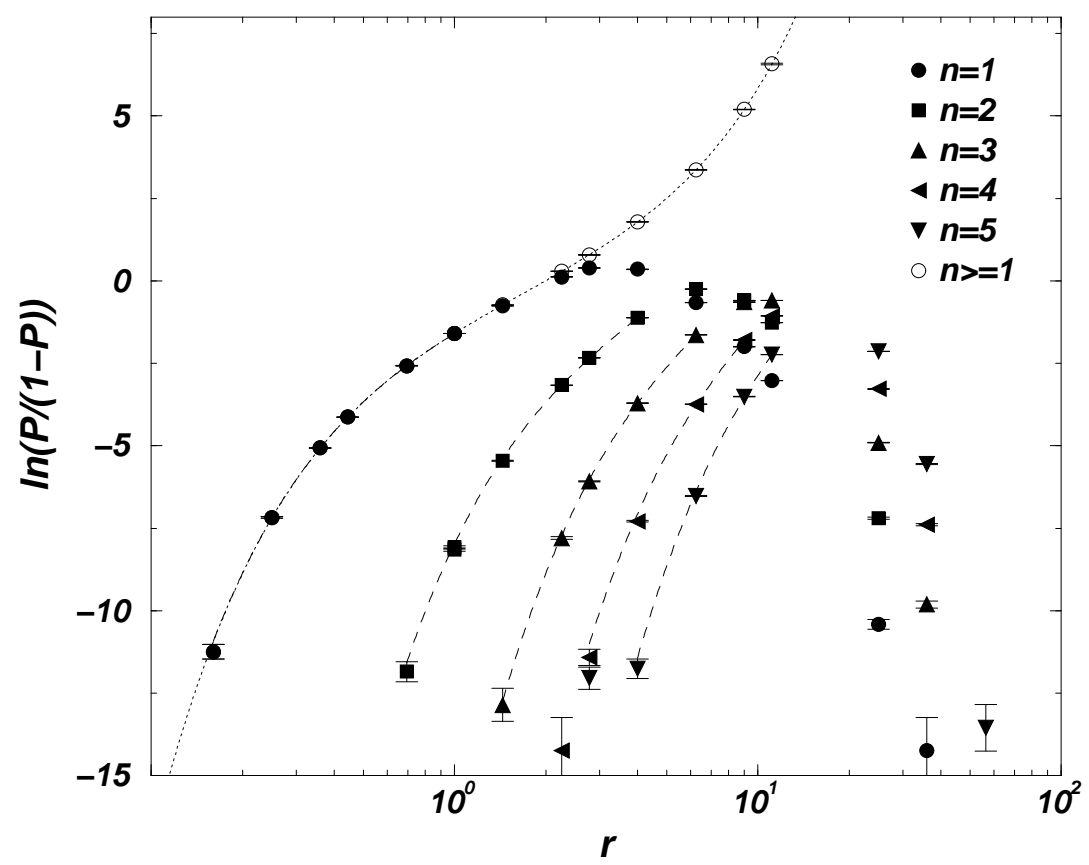

Figure 7: The rescaled probability $\mathcal{P}_{N=30000^{2}}^{(\mathrm{b})}((1,0), n, r)$ in the form $\ln (\mathcal{P} /(1-$ $\mathcal{P})$ ) for different $n$. The dotted line shows the analytical result (1), while the dashed lines are fitted according to (20) using the parameters shown in Tab. 1

The variable $C((1,0), n)$ denotes the amplitude for this type of winding and $\alpha((1,0), n)$ is expected to be a second order polynomial in $n$ 7. Clearly, the additional factor $\sqrt{r}$ is "only" a logarithmic correction to the dominating exponential, but is in fact clearly visible in the numerics.

Nothing can be derived from Section 3.4 concerning the large $r$ limit, since the average number of winding clusters and the variance thereof become very large.

Fig. 7] shows the estimated probabilities in the form $\ln (\mathcal{P} /(1-\mathcal{P}))$ [2, 14. These probabilities have been fitted against (20) in the small $r$ limit, the results of which are shown in Tab. 1 Even though the choice of (20) is somewhat arbitrary for $n>1$, very good fits are obtained. These are shown by the dashed lines in Fig. (7) which represent the fitting results of Tab. 11 fed back into (20). The main source of the numerical error is the fitting range, which is listed in the table. The range is bounded below by the smallest value of $r$ that is supported by available data within reasonable error, and bounded above by the approximate value for $r$ after which the asymptotic behavior terminates. The ambiguity of the fitting range is not reflected in the error bars, which indicate 
only the statistical error. Therefore the exact result fitted to the function (20) within the given interval should produce the values listed above.

The dotted line in Fig. [7] shows $\widehat{\mathcal{P}}((1,0), \geq 1, r)$, which asymptotically (small $r)$ contains only a single winding cluster, i.e.

$$
\lim _{r \rightarrow 0} \frac{\widehat{\mathcal{P}}((1,0), \geq 1, r)-\widehat{\mathcal{P}}((1,0), 1, r)}{\widehat{\mathcal{P}}((1,0), \geq 1, r)+\widehat{\mathcal{P}}((1,0), 1, r)}=0
$$

so that the amplitude $C((1,0), 1)$ and the exponent $\alpha((1,0), 1)$ are known exactly from (16):

$$
\begin{aligned}
C((1,0), 1) & =\sqrt{2 / 3}=0.8164909 \ldots \\
\alpha((1,0), 1) & =\pi / 2=1.5707963 \ldots
\end{aligned}
$$

which is in very good agreement with the results in Tab. 1 indicating that the fitting range chosen there is reasonable.

Just as for wrapping clusters on the cylinder, one might be tempted to find a systematic dependence of $C((1,0), n)$ and $\alpha((1,0), n)$ on $n$, such as an exponential and a second order polynomial, respectively. However, we were unable to identify these functions. Moreover, as the functional form (20) already differs from the corresponding function for wrapping clusters on the cylinder, it is not surprising that no similarities were found between their exponents and amplitudes.

\subsection{Finite size effects}

The system sizes used are so large $\left(9 \times 10^{8}\right.$ sites $)$ that one might be inclined to completely dismiss finite size corrections. Their study is nevertheless interesting for two main reasons. First, it is unknown a priori whether a system is actually large enough for finite size corrections to be neglected. Second, if they can be neglected, it is then interesting to investigate how strong the corrections are for smaller system sizes.

The strength of finite size corrections has already been discussed in [15] for a simulation with the same parameters, for crossing, spanning and wrapping on the square lattice and the cylinder. There are, apart from Pinson's exact results, no estimates for probabilities specific to winding clusters on the torus that we know of. Fig. 5 cannot be used as an indicator of high accuracy, because the aim of this paper is exactly to substantiate Pinson's analytical work and conformal invariance in percolation as such.

However, it is very instructive to compare the numerical findings for different system sizes. Most remarkably, for the cross topology, the configuration homotopic to a point, as well as winding numbers $(1,0),(1,1)$ and $(1,2)$, the distributions of the deviations from the exact results look essentially like Fig. 固 and Fig. 5 even for $N=300^{2}$. The data corresponding to the latter are shown in Fig. 8

We note that the deviations of the site percolation results from the exact values are not stronger than those of the bond percolation results. Thus we 


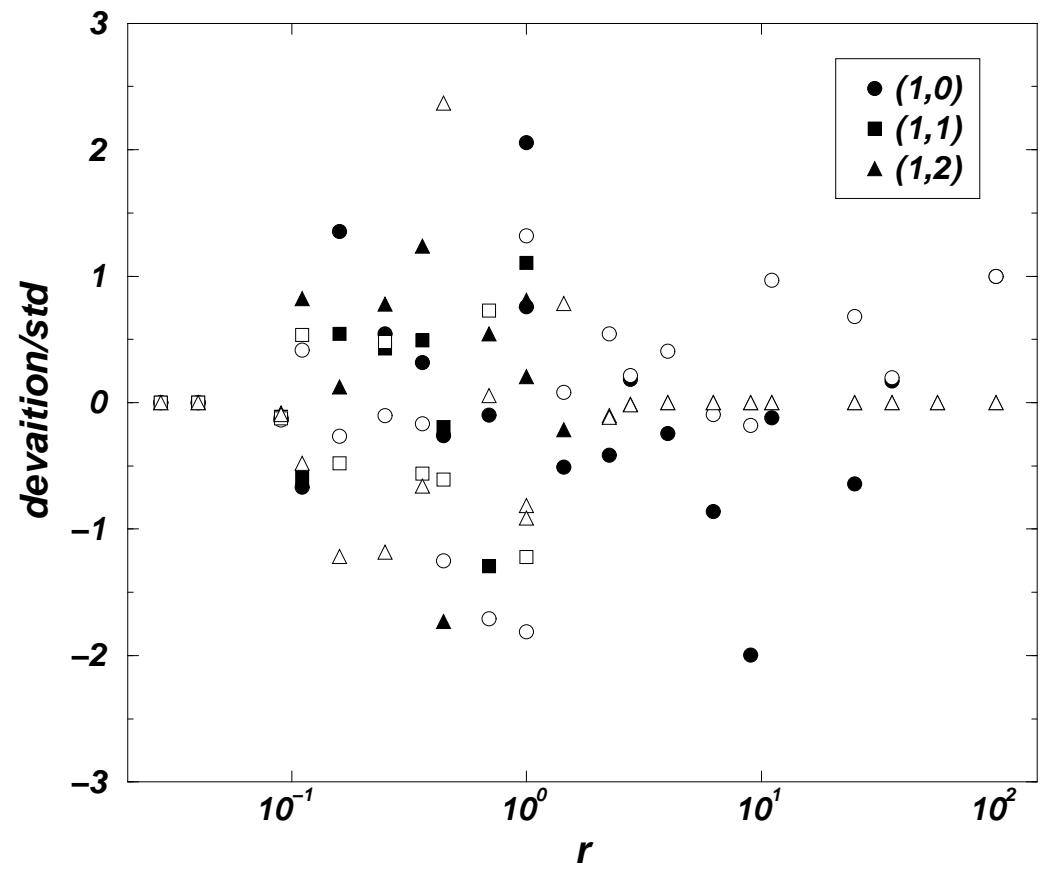

Figure 8: The deviation of $\mathcal{P}_{N=300^{2}}((a, b), \geq 1, r)$ from $\widehat{\mathcal{P}}((a, b), \geq 1, r)$ (see (11)) in units of standard deviations for $(a, b)=(1,0),(1,1),(1,2)$ and site (filled symbols) and bond (opaque symbols) percolation. There is almost no difference to the corresponding data shown for $N=30000^{2}$ in Fig. [5]

have also tested the estimate for $p^{(\mathrm{s})}=0.59274621$ [10, which was derived on much smaller system sizes.

\subsection{Möbius strip}

The probability to obtain $n$ windings on a Möbius strip is denoted in the following by $\mathcal{P}(\infty, n, r)$, where $n$ encodes the number of winding clusters and their winding numbers as discussed above. Again, two different aspects can be investigated: the probability $\mathcal{P}(\infty, \geq 1, r)$ and the probability $\mathcal{P}(\infty, a, r)$ for each individual $a=1,2, \ldots$.

Fig. 9 shows the numerical results for bond percolation and $N=30000^{2}$, again in the reduced form $\ln (\mathcal{P} /(1-\mathcal{P}))$. The behavior is qualitatively similar to the one shown for multiple wrapping clusters on the torus, Fig. 7 Using the same ansatz as for wrapping clusters on the cylinder [14,

$$
\widehat{\mathcal{P}}(\infty, n, r) \approx C(\infty, n) e^{-\alpha(\infty, n) / r},
$$

the results shown in Tab. 2 have been derived. The same precautions as in 


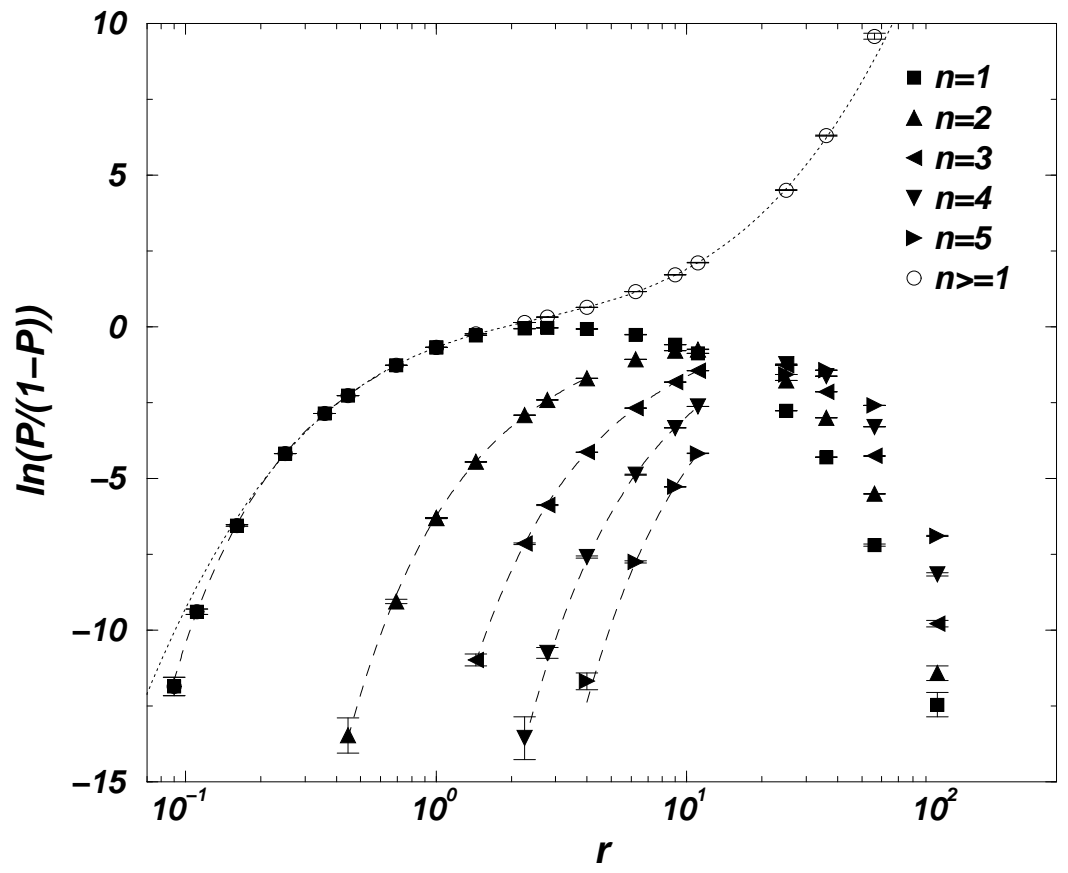

Figure 9: The rescaled probability $\mathcal{P}_{N=30000^{2}}^{(\mathrm{b})}(\infty, n, r)$ in the form $\ln (\mathcal{P} /(1-\mathcal{P}))$ for different $n$. The dashed lines show the fitting results according to (23) using the parameters shown in Tab. 2 The dotted line approximates the rescaled probability for $r$ around 1 according to (24b).

Subsec. 3.3 apply to the ranges shown in the table. While $\alpha(\infty, 1)$ can be conjectured to be $\pi / 3$ with some confidence, the functional dependence of the exponents and amplitudes on $n$ could not be found. Nevertheless, according to Fig. 9 the form (23) seems to work quite well.

In the spirit of [1] we have tried to fit $\mathcal{P}_{N=30000^{2}}(\infty, \geq 1, r)$ against a third order polynomial in $\ln (r)$. The result

$$
\begin{aligned}
& \ln \left(\frac{\mathcal{P}_{N=30000^{2}}^{(s)}(\infty, \geq 1, r)}{1-\mathcal{P}_{N=30000^{2}}^{(s)}(\infty, \geq 1, r)}\right) \approx \\
& -0.6798(7)+1.3525(9) \ln (r)-0.5648(9) \ln (r)^{2}+0.2021(3) \ln (r)^{3} \\
& \ln \left(\frac{\mathcal{P}_{N=30000^{2}}^{(\mathrm{b})}(\infty, \geq 1, r)}{1-\mathcal{P}_{N=30000^{2}}^{(\mathrm{b})}(\infty, \geq 1, r)}\right) \approx \\
& -0.6793(8)+1.3545(10) \ln (r)-0.5689(11) \ln (r)^{2}+0.2033(4) \ln (r)^{3}
\end{aligned}
$$

is shown for bond percolation in Fig. 9] as well. Similar to the results above, the 


\begin{tabular}{l|l|ll|ll}
$n$ & $r$ range & $C^{(\mathrm{s})}(\infty, n)$ & $\alpha(\infty, n)$ & $C^{(\mathrm{b})}(\infty, n)$ & $\alpha(\infty, n)$ \\
\hline 1 & $9 / 100 \ldots 25 / 36$ & $0.982(3)$ & $1.0401(14)$ & $0.985(3)$ & $1.0433(17)$ \\
2 & $25 / 36 \ldots 60 / 15$ & $0.671(2)$ & $5.832(9)$ & $0.676(3)$ & $5.849(11)$ \\
3 & $36 / 25 \ldots 100 / 9$ & $0.780(2)$ & $15.62(2)$ & $0.774(3)$ & $15.54(3)$ \\
4 & $45 / 20 \ldots 100 / 9$ & $1.081(11)$ & $30.93(10)$ & $1.099(13)$ & $31.08(12)$ \\
5 & $60 / 15 \ldots 100 / 9$ & $1.44(6)$ & $50.6(4)$ & $1.51(6)$ & $51.1(4)$
\end{tabular}

Table 2: Winding clusters on the Möbius strip; winding $n$ means $n / 2$ winding clusters with winding number 2 , if $n$ is even, and $(n-1) / 2$ clusters with winding number 2 plus a single cluster with winding number 1 , if $n$ is odd. The numerical results $\mathcal{P}_{N=30000^{2}}(\infty, n, r)$ are fitted within the range indicated against (23).

main source of error is not statistical, but systematic, namely in the choice of the specific function. Nevertheless, the numerical result (24) will possibly serve as a reference for analytical findings.

\section{Conclusion}

Based on a large scale numerical simulation, this paper provides one of the first numerical confirmations of Pinson's analytical results for winding clusters on the torus, which are based on conformal field theory. It therefore also supports conformal invariance at the critical point.

By rewriting Pinson's results, it was possible to derive some asymptotes that have hitherto only been derived for the flat topology [21, 22]. These asymptotes have been used in the investigation of the probability of multiple, simultaneously wrapping clusters.

A similar numerical analysis has been carried out for the Möbius strip, which still awaits analytical treatment.

\section{Acknowledgments}

The authors wish to thank Andy Thomas for his fantastic technical support. Without his help and dedication, this project would not have been possible. The authors also thank Dan Moore, Brendan Maguire and Phil Mayers for their continuous support. NRM is very grateful to the Beit Fellowship, and to the Zamkow family. GP gratefully acknowledges the support of the EPSRC.

\section{References}

[1] R. Langlands, C. Pichet, P. Pouliot, and Y. Saint-Aubin, J. Stat. Phys. 67, 553 (1992).

[2] J. Cardy, J. Phys. A: Math. Gen. 25, L201 (1992), preprint hep-th/9111026. 
[3] H. T. Pinson, J. Stat. Phys. 75, 1167 (1994).

[4] P. di Francesco, H. Saleur, and J. B. Zuber, J. Stat. Phys. 49, 57 (1987).

[5] C.-K. Hu and C.-Y. Lin, Phys. Rev. Lett. 77, 8 (1996).

[6] M. Aizenman, Nucl. Phys. B 485, 551 (1997).

[7] J. Cardy, J. Phys. A: Math. Gen. 31, L105 (1998).

[8] R. M. Ziff, C. D. Lorenz, and P. Kleban, Phyisica A 266, 17 (1999).

[9] M. E. J. Newman and R. M. Ziff, Phys. Rev. E 64, 016706 (2001).

[10] M. E. J. Newman and R. M. Ziff, Phys. Rev. Lett. 85, 4104 (2000).

[11] K. Kaneda and Yutaka, Phys. Rev. Lett. 86, 2134 (2001).

[12] W. T. Lu and F. Y. Wu, Phys. Rev. E 63, 026107 (2001).

[13] B. Mendelson, Introduction to Topology (Allyn and Bacon, Inc., Boston, London, Sydney, Toronto, 1975).

[14] G. Pruessner and N. R. Moloney, accepted for publication in JPA, preprint cond-mat/0309126 (unpublished).

[15] N. R. Moloney and G. Pruessner, Phys. Rev. E 67, 037701 (2003), preprint cond-mat/0211240.

[16] J. Hoshen and R. Kopelman, Phys. Rev. B 14, 3438 (1976).

[17] H. Nakanishi and H. E. Stanley, Phys. Rev. B 22, 2466 (1980).

[18] K. Binder and D. Stauffer, in Applications of the Monte Carlo Method in Statistical Physics, Vol. 36 of Topics in Current Physics, 2nd edition ed., edited by K. Binder (Springer-Verlag, Berlin Heidelberg New York, 1987), pp. $241-275$.

[19] H. Kesten, Commun. Math. Phys. 74, 41 (1980).

[20] M. Matsumoto and T. Nishimura, Monte Carlo and Quasi-Monte Carlo Methods 1998 (Springer-Verlag, Berlin Heidelberg New York, 1998), preprint from http://www.math.h.kyoto-u.ac.jp/ matumoto/RAND/DC/dc.html.

[21] R. M. Ziff, J. Phys. A: Math. Gen. 28, 1249 (1995).

[22] R. M. Ziff, J. Phys. A: Math. Gen. 28, 6479 (1995).

[23] W. Magnus, F. Oberhettinger, and R. P. Soni, Formulas and Theorems for the Special Functions of Mathematical Physics (Springer-Verlag, Berlin Heidelberg New York, 1966). 